\title{
Modulational instabilities of surface plasmons on metallic plasma surfaces with nanoparticles
}

\author{
P. K. S H U K L A ${ }^{1,2,3}$ and L. S T E N F L O O \\ ${ }^{1}$ Faculty of Physics and Astronomy, Ruhr University Bochum, \\ D-44780 Bochum, Germany \\ (ps@tp4.ruhr-uni-bochum.de) \\ ${ }^{2}$ Department of Physics, Umeå University, SE-90187 Umeå, Sweden \\ ${ }^{3}$ School of Physics, University of KwaZulu-Natal, Durban 4000, South Africa \\ ${ }^{4}$ Department of Physics (IFM), Linkoping University, Sweden
}

(Received 3 November 2007, accepted 19 November 2007, first published online 8 January 2008)

\begin{abstract}
The nonlinear coupling between high-frequency surface plasmons (SPs) and low-frequency ion oscillations on metallic plasma surfaces with charged nanoparticles is considered. It is shown that a finite-amplitude SP wave is modulationally unstable against the excitation of non-resonant ion oscillations. The growth rates and thresholds of the modulational instabilities are presented.
\end{abstract}

Heating as well as ablation of materials by intense light are of significant interest [1-3]. Intense light impinging on a metallic plasma-vacuum/dielectric interface can excite high-frequency surface plasmons (SPs) (also called surface plasma waves (SPWs)) owing to mode conversion [4] and parametric [5] processes. A largeamplitude SPW, which is an electron density wave, can ohmically heat electrons, and produce nonlinear absorption of the wave energy onto the metallic plasmavacuum/dielectric interface. Nonlinear SPWs may appear in the form of localized excitations due to a balance between the medium nonlinearity and wave dispersion. Surface plasma wave solitons occurring at the interface between a dielectric medium (air) and a nonlinear material have indeed been observed in laboratory experiments $[6,7]$. The promise $[8-10]$ of plasmonics has been recognized in the context of its potential applications in improving the resolution of microscopes as well as in enhancing the efficiency of light-emitting diodes and chemical and biological sensors/detectors. Plasmonic circuits could help the designers of computer chips to build fast interconnects that could move large amounts of data across the chips.

In the present letter, we consider the amplitude modulation of a large-amplitude SPW that is propagating along a metallic plasma-vacuum/dielectric surface coated with charged nanoparticles. In such a condensed matter Fermi plasma, a quantum force associated with the Bohm potential [11] acts on the electrons, in addition to the electromagnetic forces. Thus, the dispersion properties of the high-frequency SPWs and low-frequency ion oscillations (IOs) are significantly affected by the quantum force. The SPWs interacting nonlinearly with the IOs would generate SP sidebands. The latter, in turn, would interact with the SP pump to produce a low-frequency ponderomotive force, which can eventually reinforce the IOs. As a result, there appear modulational instabilities [12] due to which the sidebands and 
the IOs grow at the expense of the SP pump energy. The modulational instability may be responsible for the formation of envelope solitons [13-15].

The dynamics of nonlinearly coupled SPWs and IOs on a metallic plasmavacuum/dielectric surface is governed by the continuity equation

$$
\frac{\partial n_{j 1}}{\partial t}+n_{j 0} \nabla \cdot \mathbf{v}_{j}=0
$$

the momentum equation

$$
m_{j}\left(\frac{\partial}{\partial t}+\mathbf{v}_{j} \cdot \nabla\right) \mathbf{v}_{j}=q_{j} \mathbf{E}-\frac{k_{\mathrm{B}} T_{\mathrm{F} j}}{n_{j 0}} \nabla n_{j 1}+\frac{\hbar^{2}}{4 m_{j} n_{j 0}} \nabla \nabla^{2} n_{j 1},
$$

and the Poisson equation

$$
\nabla \cdot \mathbf{E}=4 \pi\left(q_{\mathrm{i}} n_{\mathrm{i} 1}+q_{\mathrm{e}} n_{\mathrm{e} 1}\right),
$$

where $n_{j 1}\left(\ll n_{j 0}\right)$ is a perturbation of the density of the particle species $j$ ( $j$ equals e for electrons and i for ions), $n_{j 0}$ is the unperturbed particle number density, $\mathbf{v}_{j}$ is the fluid velocity perturbation, $\mathbf{E}=-\nabla \phi$ is the electrostatic field, $\phi$ is the electrostatic potential, $q_{j}=-e\left(Z_{\mathrm{i}} e\right)$ for the electrons (ions), $e$ is the magnitude of the electron charge, $Z_{\mathrm{i}}$ is the ion charge state, $m_{j}$ is the mass, $k_{\mathrm{B}}$ is the Boltzmann constant, and $T_{\mathrm{F} j}$ is the Fermi temperature (in fact, for a condensed Fermi plasma, we have [16] $k_{\mathrm{B}} T_{\mathrm{F} j}=\left(\hbar^{2} / 2 m_{j}\right)\left(3 \pi^{2}\right)^{1 / 3} n_{j 0}^{2 / 3}$, where $\hbar$ is the Planck constant divided by $\left.2 \pi\right)$. The third term on the right-hand side of $(2)$ is the quantum force associated with the Bohm potential [11], which can cause electron tunneling. At equilibrium, we have $Z_{\mathrm{i}} n_{\mathrm{i} 0}=n_{\mathrm{e} 0}+Z_{\mathrm{d}} n_{\mathrm{d} 0}$, where $Z_{\mathrm{d}}$ is the number of electrons residing on nanoparticles. The latter are supposed to be immobile, since we are considering surface wave phenomena on time scales much shorter than the nanoparticle plasma period.

The frequency $\omega$ of the SPW on the metallic plasma-vacuum interface can be obtained from (1)-(3) by supposing that the ions do not participate in the SPW dynamics. Following the general approach of [17], we obtain

$$
\omega \approx \frac{\omega_{\mathrm{pe}}}{\sqrt{2}}\left(1+\frac{k V_{\mathrm{Fe}}}{\sqrt{2} \omega_{\mathrm{pe}}} \sqrt{1+\hbar^{2} k^{2} / 4 m_{\mathrm{e}}^{2} V_{\mathrm{Fe}}^{2}}\right)
$$

where $k$ is the wavenumber (along the direction of the plasma-vacuum/dielectric interface), $\omega_{\mathrm{pe}}=\left(4 \pi n_{\mathrm{e}} e^{2} / m_{\mathrm{e}}\right)^{1 / 2}$ is the electron plasma frequency, and $V_{\mathrm{Fe}}=$ $\left(k_{\mathrm{B}} T_{\mathrm{Fe}} / m_{\mathrm{e}}\right)^{1 / 2}$ is the Fermi thermal speed. In the limit $\hbar k / 2 m_{\mathrm{e}} V_{\mathrm{Fe}} \gg 1$, we have from (4)

$$
\omega \approx \frac{\omega_{\mathrm{pe}}}{\sqrt{2}}\left(1+\frac{\hbar k^{2}}{2 \sqrt{2} m_{\mathrm{e}} \omega_{\mathrm{pe}}}\right) \equiv \frac{\omega_{\mathrm{pe}}}{\sqrt{2}}(1+\beta) .
$$

The group velocity and the group dispersion of the SPWs, given by (5), are $V_{\mathrm{g}}=$ $\partial \omega / \partial k=\hbar k / 2 m_{\mathrm{e}}$ and $\partial V_{\mathrm{g}} / \partial k=\hbar / 2 m_{e}$, respectively.

We now consider the amplitude modulation of the SPWs, given by (5). Due to the nonlinear coupling between the SPW pump and the IOs, one encounters an envelope of the SPWs whose electric field varies slowly in space and time. The equation governing the envelope of the SPW in the presence of the IOs is

$$
i\left(\frac{\partial}{\partial \tau}+V_{\mathrm{g}} \frac{\partial}{\partial \xi}\right) E_{z}+P \frac{\partial^{2} E_{z}}{\partial \xi^{2}}-\frac{\omega_{\mathrm{p}}}{2 \sqrt{2}} \frac{n_{\mathrm{es}}}{n_{\mathrm{e} 0}} E_{z}=0,
$$

where $\partial E_{z} / \partial \tau \ll \omega E_{z}, \partial E_{z} / \partial \xi \ll k E_{z}, E_{z}$ is the SPW electric field along the $\xi$-direction at the surface, $P \approx \hbar / 4 m_{\mathrm{e}}$, and $\omega_{\mathrm{p}}=\left(4 \pi n_{\mathrm{e} 0} e^{2} / m_{\mathrm{e}}\right)^{1 / 2}$ is the unperturbed 
plasma frequency. The slowly varying time and space variables in (6) are denoted here by $\tau$ and $\xi$, respectively. Furthermore, the electron number density perturbation associated with the low-phase speed IOs is denoted by $n_{\mathrm{es}}\left(\ll n_{\mathrm{e} 0}\right)$. It is obtained from the inertialess electron momentum

$$
\frac{e^{2}}{m_{\mathrm{e}} \omega_{\mathrm{p}}^{2}(1+\beta)^{2}} \frac{\partial\left|E_{z}\right|^{2} \exp (-2 k x)}{\partial \xi}=e \frac{\partial \varphi}{\partial \xi}+\frac{\hbar^{2}}{4 m_{\mathrm{e}} n_{\mathrm{e} 0}} \frac{\partial^{3} n_{\mathrm{es}}}{\partial^{3} \xi},
$$

where $x$ is the direction normal to the surface, and $\varphi$ is the electrostatic potential associated with the IOs. The left-hand side of (7) represents the ponderomotive force of the SPW. We note that (7) is valid for $\partial^{2} n_{\mathrm{es}} / \partial \tau^{2} \ll\left(\hbar^{2} / 4 m_{\mathrm{e}}^{2}\right) \partial^{4} n_{\mathrm{es}} / \partial \xi^{4}$ and $k_{\mathrm{B}} T_{\mathrm{Fe}} n_{\mathrm{es}} \ll\left(\hbar^{2} / 4 m_{\mathrm{e}}\right) \partial^{2} n_{\mathrm{es}} / \partial \xi^{2}$, so that the electron inertial and the electron pressure gradient are neglected, respectively.

The electrons are coupled to the ions via the electrostatic potential $\varphi$. The equations governing the ion dynamics supporting IOs are

$$
\frac{\partial n_{\mathrm{is}}}{\partial \tau}+n_{\mathrm{i} 0} \frac{\partial u}{\partial \xi}=0
$$

and

$$
m_{\mathrm{i}} \frac{\partial u}{\partial \tau}=-e \frac{\partial \varphi}{\partial \xi}
$$

where $n_{\text {is }}\left(\ll n_{\text {i0 }}\right)$ is the ion number density perturbation, $u$ is the ion fluid velocity, and $m_{\mathrm{i}}$ is the ion mass. The ion quantum force and the ponderomotive force of the SPWs acting on the ion fluid are smaller by a factor $m_{\mathrm{e}} / m_{\mathrm{i}}$ (in comparison to that on the electrons), and therefore ignored in (9).

We now combine (7)-(9) by using the quasi-neutrality condition $n_{\mathrm{es}}=n_{\text {is }}$, to obtain the wave equation for the IOs [18] in the presence of the SPWs,

$$
\left(\frac{\partial^{2}}{\partial \tau^{2}}+\frac{\alpha \hbar^{2}}{4 m_{\mathrm{e}} m_{\mathrm{i}}} \frac{\partial^{4}}{\partial \xi^{4}}\right) \frac{n_{\mathrm{es}}}{n_{\mathrm{e} 0}}=\alpha \frac{\exp (-2 k x)}{4 \pi n_{\mathrm{e} 0} m_{\mathrm{i}}(1+\beta)^{2}} \frac{\partial^{2}\left|E_{z}\right|^{2}}{\partial \xi^{2}},
$$

where $\alpha=n_{\mathrm{i} 0} / n_{\mathrm{e} 0}$. We see that the effect of charged nanoparticles appears through $\alpha$, which is larger than unity in our plasma.

Equations (6) and (10) are the desired equations for investigating the modulational instability of a constant-amplitude SP pump. Following the standard technique [19] of parametric instability investigations, we then obtain the nonlinear dispersion relation

$$
\left(\Omega^{2}-\Omega_{q}^{2}\right)\left[\left(\Omega-K V_{\mathrm{g}}\right)^{2}-P^{2} K^{4}\right]=\alpha P K^{4} \frac{\omega_{\mathrm{p}}\left|E_{z 0}\right|^{2} \exp (-2 k x)}{4 \sqrt{2} \pi n_{\mathrm{e} 0} m_{\mathrm{i}}(1+\beta)^{2}}
$$

where $\Omega_{q}=\sqrt{\alpha} \hbar K^{2} / 2 \sqrt{m_{\mathrm{e}} m_{\mathrm{i}}}, \Omega$ and $K$ are the frequency and the wavenumber of the IOs, respectively, and $E_{z 0}$ is the electric field of the SP pump.

Two comments are in order. First, for $\Omega \gg K V_{\mathrm{g}}$, we have from (11)

$$
\Omega^{4}-\Omega^{2}\left(\Omega_{q}^{2}+P^{2} K^{4}\right)+\Omega_{q}^{2} P^{2} K^{4}-\alpha P K^{4} \frac{\omega_{\mathrm{p}}\left|E_{z 0}\right|^{2} \exp (-2 k x)}{4 \sqrt{2} \pi n_{\mathrm{e} 0} m_{\mathrm{i}}(1+\beta)^{2}}=0,
$$

which has the solutions

$$
\Omega^{2}=\frac{1}{2}\left(\Omega_{q}^{2}+P^{2} K^{4}\right) \pm \frac{1}{2}\left[\left(\Omega_{q}^{2}-P^{2} K^{4}\right)^{2}+\alpha P K^{4} \frac{\omega_{\mathrm{p}}\left|E_{z 0}\right|^{2} \exp (-2 k x)}{\sqrt{2} \pi n_{\mathrm{e} 0} m_{\mathrm{i}}(1+\beta)^{2}}\right]^{1 / 2} .
$$


Equation (13) admits a modulational instability, and the growth rate is obtained by letting $\Omega=\Omega_{\mathrm{r}}+i \Omega_{\mathrm{i}}$, where $\Omega_{\mathrm{r}}$ and $\Omega_{\mathrm{i}}$ are the real and imaginary parts of the modulation frequency. Second, for $\Omega \ll \Omega_{q}$, (11) reduces to

$$
\left(\Omega-K V_{\mathrm{g}}\right)^{2}=P K^{4}\left[P-\alpha \frac{\omega_{\mathrm{p}}\left|E_{z 0}\right|^{2} \exp (-2 k x)}{4 \sqrt{2} \pi n_{\mathrm{e} 0} m_{\mathrm{i}}(1+\beta)^{2} \Omega_{q}^{2}}\right],
$$

which predicts an oscillatory modulational instability if

$$
\left|E_{z 0}\right|^{2} \exp (-2 k x)>\frac{\sqrt{2} \pi m_{\mathrm{i}} n_{\mathrm{e} 0} \hbar \Omega_{q}^{2}(1+\beta)^{2}}{\alpha m_{\mathrm{e}} \omega_{\mathrm{p}}} .
$$

To summarize, we have considered the nonlinear coupling between finiteamplitude SPWs and IOs at metallic plasma-vacuum/dielectric surfaces with charged nanoparticles. It is shown that such nonlinear interactions lead to modulational instabilities via which the SPW sidebands and the IOs grow on account of the SPW pump energy. We expect that modulationally unstable waves will evolve in the form of envelope SPW solitons. The latter are localized excitations, which can transport the wave energy at metallic plasma surfaces having charged nanoparticles.

\section{Acknowledgements}

This research was partially supported by the Deutsche Forschungsgemeinschaft through the Sonderforschungsbereich 591 entitled 'Universelles Verhalten Gleichgewichtsferner Plasmen: Heizung, Transport und Strukturbildung'.

\section{References}

[1] Raether, H. 1988 Surface Plasmons on Smooth and Rough Surfaces and on Gratings (Springer Tracts in Modern Physics, 11). New York: Springer.

[2] Shalaev, V. M. 2007 Nature Photonics (London) 1, 41.

[3] Kumar, G. and Tripathi, V. K. 2007 Appl. Phys. Lett. 91, 161503.

[4] Liu, C. S., Kumar, G. and Tripathi, V. K. 2006 J. Appl. Phys. 100, 013304.

[5] Kumar, N. and Tripathi, V. K. 2007 Phys. Plasmas 14, 103108.

[6] Wang, X. et al. 2007 Phys. Rev. Lett. 98, 123903.

[7] Alfassi, B. et al. 2007 Phys. Rev. Lett. 98, 213901.

[8] Barnes, W. L., Dereux, A. and Ebbesen, T. W. 2003 Nature (London) 424, 824.

[9] Atwater, H. A. 2007 The Promise of Plasmonics, Scientific American (Scientificamerican.com), $\mathbf{2 9 6}(4), 00368733$.

[10] Diaconsu, B. et al. 2007 Nature (London) 448, 57.

[11] Gardner, C. L. and Ringhofer, C. 1996 Phys. Rev. E 53, 157. Manfredi, G. and Haas, F. 2001 Phys. Rev. B 64, 075316. Shukla, P. K. and Eliasson, B. 2006 Phys. Rev. Lett. 96, 245001. Shukla, P. K. and Eliasson, B. 2007 Phys. Rev. Lett. 99, 096401.

[12] Yu, M. Y., Spatschek, K. H. and Shukla, P. K. 1974 Z. Naturforsch. 29, 1736. Shukla, P. K., Yu, M. Y. and Spatschek, K. H. 1975 Phys. Fluids 18, 265. Sharma, R. P. and Shukla, P. K. 1983 Phys. Fluids 26, 87. Shukla, P. K. and Stenflo, L. 1984 Phys. Rev. A 30, 2110. Murtaza, G. and Shukla, P. K. 1984 J. Plasma Phys. 31, 423.

[13] Kaufman, A. N. and Stenflo, L. 1975 Phys. Scr. 11, 269. Schamel, H., Yu, M. Y. and Shukla, P. K. 1977 Phys. Fluids 20, 1286. Yu, M. Y. and Shukla, P. K. 1977 Plasma Phys. 19, 889. 
[14] Yu, M. Y. and Zhelyazkov, I. 1978 J. Plasma Phys. 20, 183.

[15] Gradov, O. M. and Stenflo, L. 1983 Phys. Rep. 94, 111.

Gradov, O. M. and Stenflo, L. 1991 Phys. Fluids B 3, 3201.

Vladimirov, S. V., Yu, M. Y. and Stenflo, L. 1993 Phys. Lett. A 174, 313.

[16] Manfredi, G. 2005 Fields Inst. Commun. 46, 263.

Shaikh, D. and Shukla, P. K. 2007 Phys. Rev. Lett. 99, 125002.

[17] Kaw, P. K. and McBride, J. B. 1970 Phys. Fluids 13, 1784.

[18] Shukla, P. K. and Stenflo, L. 2006 Phys. Plasmas 13, 044505.

[19] Shukla, P. K., Rao, N. N., Yu, M. Y. and Tsintsadze, N. L. 1986 Phys. Rep. 138, 1.

Marklund, M. and Shukla, P. K. 2006 Rev. Mod. Phys. 78, 591. 